\title{
Novel drug discovery system for cancer stem cells in human squamous cell carcinoma of the esophagus
}

\author{
YOSHIHIRO KANO ${ }^{1,2^{*}}$, MASAMITSU KONNO ${ }^{2 *}$, KOICHI KAWAMOTO ${ }^{1,2}$, KEISUKE TAMARI $^{3}$ \\ KAZUHIKO HAYASHI ${ }^{3}$, TAKAHITO FUKUSUMI ${ }^{4}$, TAROH SATOH ${ }^{2}$, SHINJI TANAKA $^{5}$, \\ KAZUHIKO OGAWA ${ }^{3}$, MASAKI MORI ${ }^{1}$, YUICHIRO DOKI ${ }^{1}$ and HIDESHI ISHII ${ }^{2}$ \\ Departments of ${ }^{1}$ Gastroenterological Surgery, ${ }^{2}$ Frontier Science for Cancer and Chemotherapy, \\ ${ }^{3}$ Radiation Oncology and ${ }^{4}$ Otorhinolaryngology-Head and Neck Surgery, Osaka University Graduate \\ School of Medicine, Suita, Osaka 565-0871; ${ }^{5}$ Department of Hepato-Biliary-Pancreatic Surgery, \\ Graduate School of Medicine, Tokyo Medical and Dental University, Tokyo 113-0034, Japan
}

Received October 10, 2013; Accepted November 4, 2013

DOI: $10.3892 /$ or.2013.2952

\begin{abstract}
Cancer stem cells (CSCs) have been identified in several tumor tissues. Since CSCs are resistant to cancer therapies, including chemotherapy and radiation therapy, and can even remain after therapies, tumor tissue often regrows and relapses. Thus, identification of CSCs and treatment targeting CSCs are required to treat tumor tissues. Reportedly, a fluorescent vector consisting of fluorescein ZsGreen fused to the carboxyl-terminal region of ornithine decarboxylase (cODC) was used to detect CSCs or therapy-resistant cancer cells in tumor tissues of the brain, pancreas and liver. Cells transfected with the fluorescent vector can express a fluorescein fused to cODC and become fluorescent only when the fusion protein is accumulated. In the present study, CSCs or therapy-resistant cancer cells were identified with the fluorescent vector in esophageal squamous cell carcinoma. The use of this fluorescent vector in drug screening enabled the detection of three drugs, AKT inhibitor XI, ERK inhibitor II and JAK inhibitor I, which target malignant CSCs.
\end{abstract}

\section{Introduction}

Esophageal squamous cell carcinoma (SCC) is a fatal disease that frequently occurs in Asia and South America (1). Cancer stem cells (CSCs) exhibit several characteristics that make cancerous tumors fatal. CSCs produce several types of prolif-

Correspondence to: Professor Hideshi Ishii, Department of Frontier Science for Cancer and Chemotherapy, Osaka University Graduate School of Medicine, 2-2 Yamadaoka, Suita, Osaka 565-0871, Japan E-mail: hishii@gesurg.med.osaka-u.ac.jp

*Contributed equally

Key words: ornithine decarboxylase, esophageal cancer, novel drug discovery erative progenies, are resistant to several drugs and radiation and are tumorigenic. Due to CSCs, tumor tissue often regenerates after therapy. CSCs have been detected in several types of tumor tissues such as breast cancer and pancreas adenocarcinoma $(2,3)$. Thus, CSCs are thought to have critical roles in therapy resistance, relapse and metastasis as well. Recently, some CSCs in SCC were detected. CD75, CD44, CD90 and side population cells were reported to be CSC markers (4-7). Moreover, in our previous study, JARID1B was used as a CSC marker (8). However, therapies for esophageal SCC that target CSCs remain relatively unknown.

In the present study, we used a fluorescent vector that can detect CSCs or therapy-resistant cells in some tumor tissues (9-13). The vector is a fluorescein ZsGreen fused to the carboxyl-terminal degron of ornithine decarboxylase (cODC). The cells infected with the vector become ZsGreenpositive when the protein is not degradated and become ZsGreen-negative when the protein is lysed. On the basis of this mechanism, this protein is believed to be able to detect the function of protein degradation machineries, such as proteasomes. In the present study, we investigated the use of the above-mentioned fluorescent vector for detection of CSCs or therapy-resistant cells in esophageal SCC.

\section{Materials and methods}

Cell culture. The human esophageal SCC cell lines TE4 and TE8 were cultured in RPMI-1640 medium supplemented with $10 \%$ fetal bovine serum (FBS; HyClone, Logan, UT, USA) at $5 \% \mathrm{CO}_{2}$ and $37^{\circ} \mathrm{C}$. The Platinum-A Retroviral Packaging Cell Line (Plat-A) was purchased from Cell Biolabs (San Diego, CA, USA). Plat-A was cultured in DMEM supplemented with 10\% FBS, $1 \mu \mathrm{g} / \mathrm{ml}$ puromycin (Sigma-Aldrich, St. Louis, MO, USA), $10 \mu \mathrm{g} / \mathrm{ml}$ blasticidin (Sigma-Aldrich), $100 \mathrm{U} / \mathrm{ml}$ penicillin and streptomycin (Life Technologies, Inc., Gaithersburg, MD, USA). StemPro Accutase (Life Technologies) was used for detachment of all the cells. A retroviral vector known as pQCXIN-ZsGreen-cODC consisting of a fluorescein ZsGreen fused to cODC was kindly provided by Dr Shinji Tanaka. The 
A

\section{ZsGreen $O D C$ degron sequence}

B

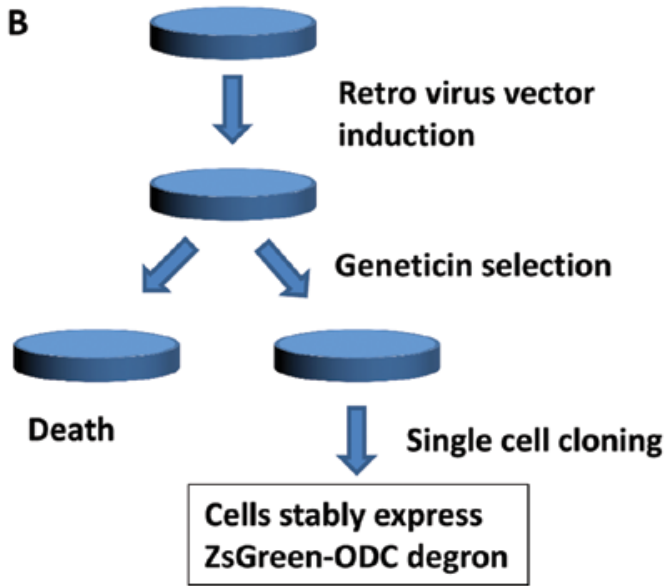

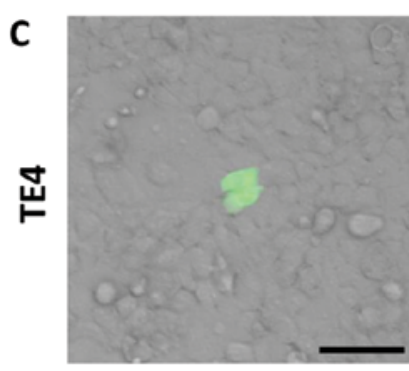

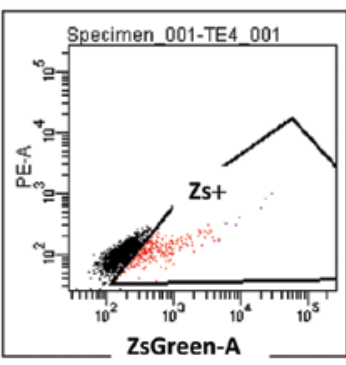

D

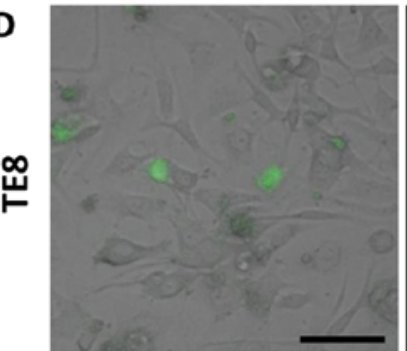

Figure 1. Establishment of visualized CSC-like cells. (A) Presented is a part of the retroviral vector, which consists of fluorescein ZsGreen fused to cODC. (B) Stable construction of cells expresses a fluorescent fusion protein. (C and D) Fluorescent cells are detected by microscopy (left panels) and by FACS analysis (right panels) in the esophageal SCC cell lines TE4 (C) and TE8 (D). Scale bar, $100 \mu \mathrm{m}$.

retroviral vector was transfected into Plat-A to generate retrovirus. The virus collected from the supernatant of the Plat-A was used to induct cells. The cells stably expressing fluorescein ZsGreen fused to cODC were selected by adding $500 \mu \mathrm{g} / \mathrm{ml}$ of G418 (Life technologies) and purified by single-cell cloning.

Sphere formation. The ReproStem medium (ReproCELL, Inc., Kanagawa, Japan) supplemented with $5 \mathrm{ng} / \mathrm{ml}$ fibroblast growth factor 2 (ReproCELL) was used to suspend $1 \times 10^{3}$ cells. Subsequently, $1 \times 10^{3}$ cells were seeded in ultra-low attachment 6 -well plates (Corning Incorporated, Corning, NY, USA). Following incubation for $\sim 2$ weeks, formed spheres $>100 \mu \mathrm{m}$ in size were counted.

Clonogenic survival assay. Appropriate numbers of cells were seeded in 10-cm dishes and exposed to radiation at 0,2, 4 and 6 Gy. Following incubation for $\sim 2$ weeks, colonies stained with the Diff-Quick solution (Sysmex, Kobe, Japan) were counted.

Drug screening. Cell Counting Kit-8 (Dojindo Laboratories, Kumamoto, Japan), in which $2 \times 10^{3}$ cells/100 $\mu$ l were seeded into each well of a 96 -well plate, was used to determine cell viability. Following incubation for $24 \mathrm{~h}$, the cells were exposed to drugs or radiation. Then, following incubation for $72 \mathrm{~h}$, $10 \mu \mathrm{l}$ of Cell Counting Kit-8 solution was added to each well followed by further incubation for $2 \mathrm{~h}$. Cell viability was determined by reading the optical density (Bio-Rad Laboratories, Hercules, CA, USA) in each well at $450 \mathrm{~nm}$.

Animal experiments. Portions containing $1 \times 10^{3}$ cells, mixed with BD Matrigel (Becton-Dickinson, Franklin Lakes, NJ, USA) at a 1:1 ratio, were subcutaneously injected into NOD/SCID mice. These mice were examined for 51 days and sacrificed when the tumors reached a maximum diameter of $\sim 15 \mathrm{~mm}$. The animal studies were approved by the Animal Experiments Committee of Osaka University (Suita, Japan).

\section{Results}

Establishment of visualized CSC-like cells of human esophagus. Two esophageal SCC cell lines, TE4 and TE8, were infected with a retroviral vector containing fluorescein ZsGreen fused to cODC (the fusion protein is presented in Fig. 1A). Following Geneticin ${ }^{\circledR}$ selection and single-cell cloning, the cells stably expressing fluorescein ZsGreen fused to cODC were generated (Fig. 1B). The ZsGreen-positive cells were detected by microscopy and FACS analysis in both cell lines. Approximately $15 \%$ of the population was detected as ZsGreen-positive by FACS analysis, but only $\sim 0.1 \%$ of the population was detected as ZsGreen-positive by microscopy in both the cell lines (Fig. 1C and D).

Self-renewal study. To investigate the characteristics of the ZsGreen-high cells and the ZsGreen-negative cells, a sphereforming assay was performed. A sphere-forming assay is often performed to investigate the self-renewal capacity of cells, which is one of the characteristics of CSCs. An FACS sorter was used to isolate the ZsGreen-high and ZsGreen-negative cells, and $1 \times 10^{3}$ cells/well were seeded into low-attachment 6 -well plates. Following incubation for $\sim 2$ weeks, formed spheres $>100 \mu \mathrm{m}$ in size were counted. As a result, both the ZsGreen-high and ZsGreen-negative cells formed in TE4 (Fig. 2A and B) and TE8 (Fig. 2D and E), but differences were observed in the number of formed spheres between these two populations. More spheres were formed by the ZsGreen-high cells than by the ZsGreen-negative cells in TE4 (Fig. 2C) and TE8 (Fig. 2F) cells.

Sensitivity to chemotherapy agents. To further investigate the characteristics of the ZsGreen-high and ZsGreen-negative cells, the drug sensitivities of these two populations were analyzed. In the present study, cisplatin (CDDP) and fluorouracil (5-FU) were used. These drugs are often used to treat 

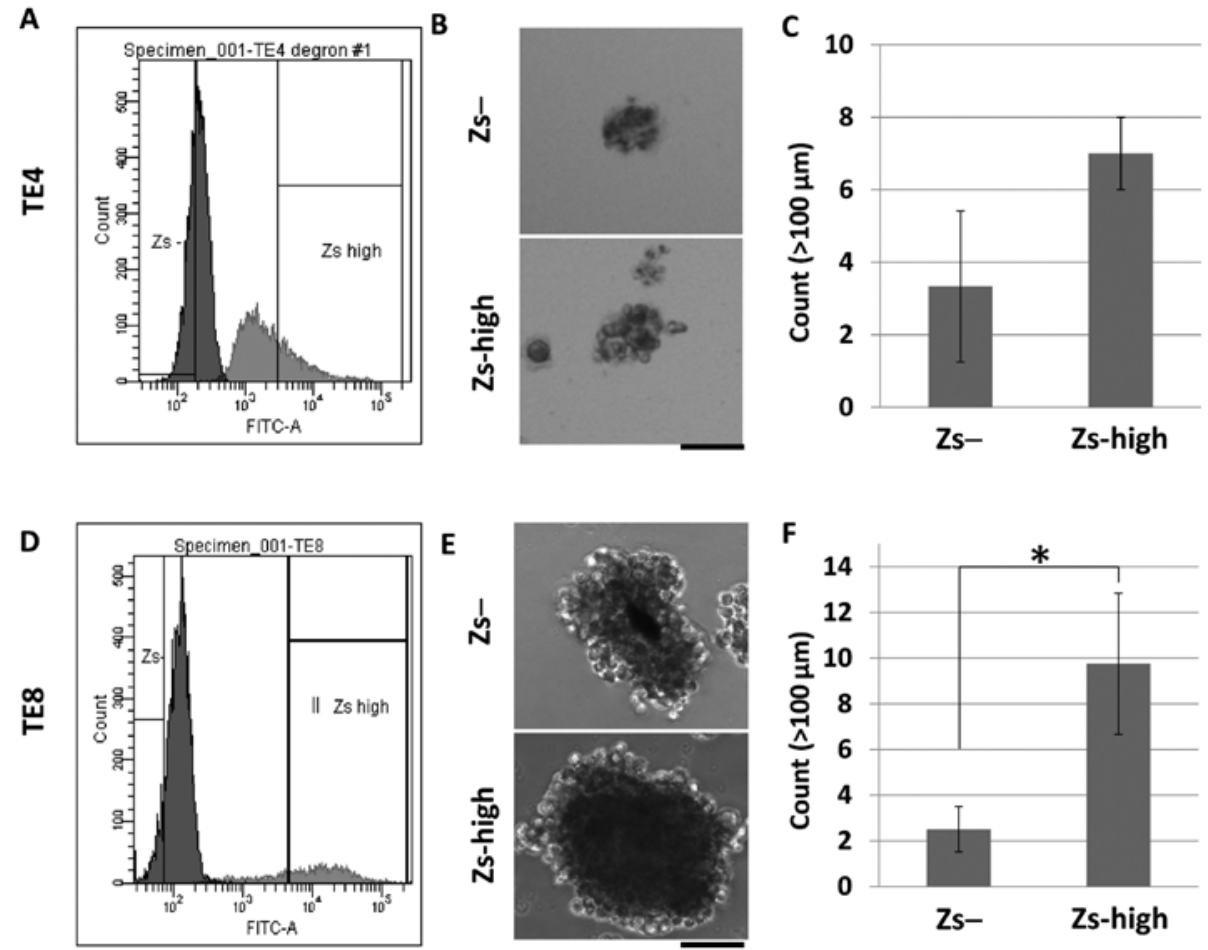

Figure 2. Self-renewal study of the fluorescent and non-fluorescent cells. (A and D) ZsGreen-high (gated in P6) and ZsGreen-negative cells (gated in P7) isolated by FACS sorting in TE4 (A) and TE8 (D) cells. (B and E) The spheres formed after incubation for 1 week in TE4 (B) and TE8 (E) cells are displayed. (C and F) Presented are the number of spheres formed with the ZsGreen-high and ZsGreen-negative cells in TE4 (C) and TE8 (F) cells. The error bars represent the standard deviation of the mean for three experiments; asterisks indicate that the mean is significantly different between ZsGreen-high cells and ZsGreen-negative cells (Student's t-test; "P<0.05). Scale bar, $100 \mu \mathrm{m}$.
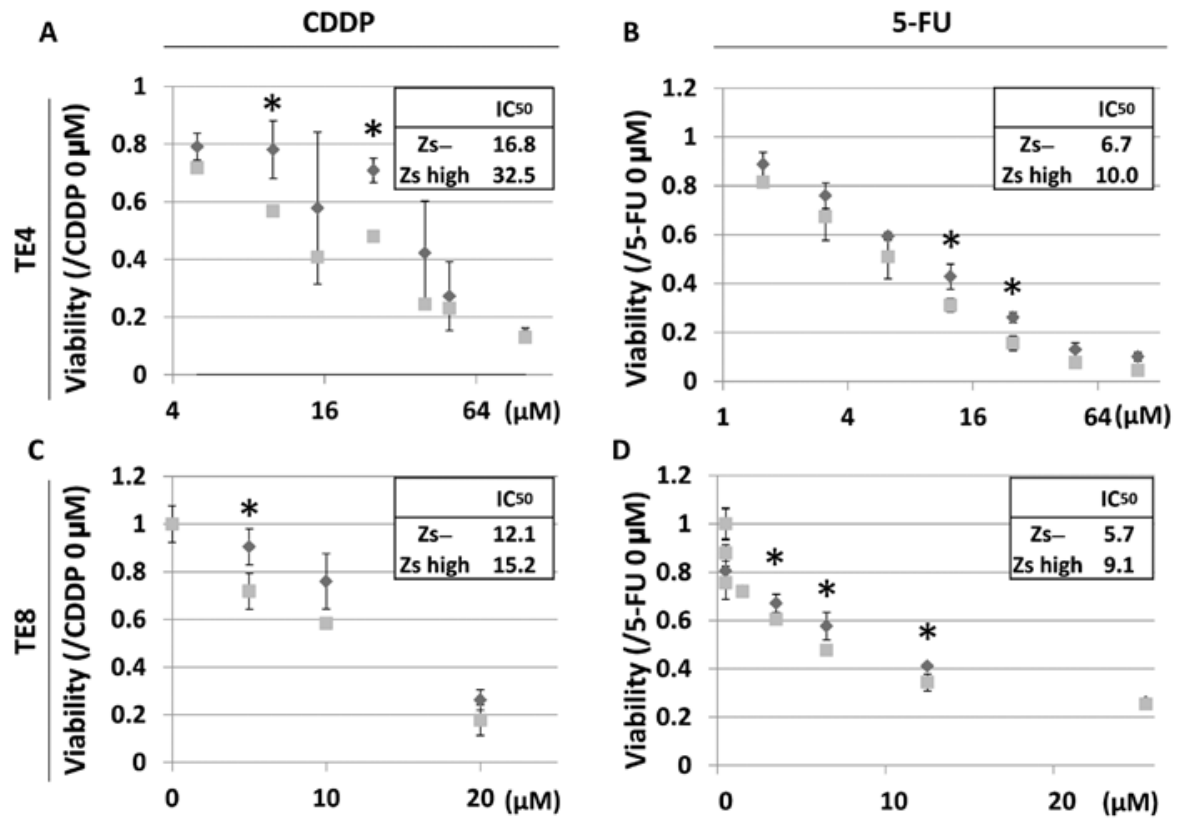

Figure 3. Drug sensitivity of the fluorescent and non-fluorescent cells. Survival curves of TE4 and TE8 cells treated with CDDP (A and C) and with 5-FU (B and D) in the ZsGreen-high and ZsGreen-negative cells after incubation with increasing concentrations for $72 \mathrm{~h}$ in TE4 (A and B) and TE8 (C and D) cells. The error bars represent the standard deviation of the mean for three experiments; asterisks indicate that the mean is significantly different between ZsGreen-high cells and ZsGreen-negative cells (Student's t-test; ${ }^{\mathrm{P}}<0.05$ ).

esophageal SCCs. As a result, the $\mathrm{IC}_{50}$ values of the two drugs were higher for the ZsGreen-high cells compared with those for the ZsGreen-negative cells (Fig. 3).

Sensitivity to ionized radiation. Since radiotherapy is often used to treat esophageal SCCs as well, the radiation sensi- tivities of the ZsGreen-high and ZsGreen-negative cells were analyzed. First, the viabilities of these two populations after exposure to radiation were analyzed. The FACS sorter was used to isolate these two populations, which were then seeded into 96-well plates and exposed to radiation at 0 and $8 \mathrm{~Gy}$. Following incubation for $120 \mathrm{~h}$, the viabilities were analyzed. 

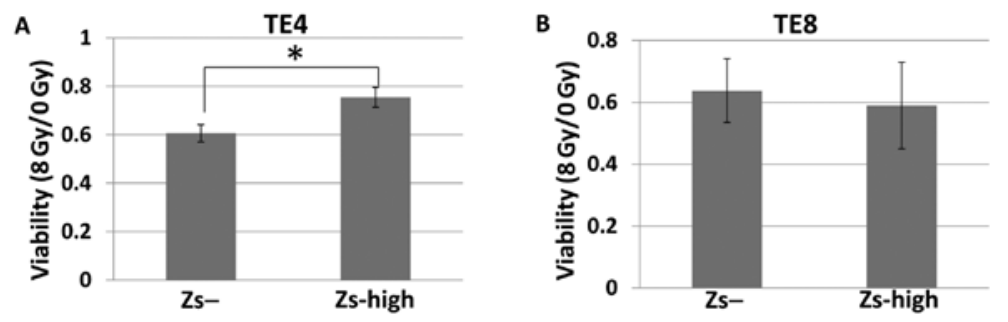

\begin{tabular}{|c|c|c|}
\hline \multirow{2}{*}{$\begin{array}{c}\text { Gy } \\
\text { (seeded number } \\
\text { of cells) }\end{array}$} & \multicolumn{2}{|c|}{$\begin{array}{c}\text { Number of } \\
\text { colonies }\end{array}$} \\
\cline { 2 - 3 } $\begin{array}{c}\text { 0 Gy } \\
\text { (400 cells) }\end{array}$ & 65 & 91 \\
\cline { 2 - 3 } & 62 & 90 \\
\cline { 2 - 3 } 2 2 Gy & 63 & 66 \\
\cline { 2 - 3 } (400 cells) & 11 & 42 \\
\cline { 2 - 3 } & 29 & 36 \\
\hline \multirow{2}{*}{4 Gy } & 9 & 24 \\
\cline { 2 - 3 } (800 cells) & 11 & 21 \\
\cline { 2 - 3 } & 14 & 32 \\
\hline \multirow{2}{*}{6 Gy } & 2 & 16 \\
\cline { 2 - 3 } (2000 cells) & 5 & 16 \\
\cline { 2 - 3 } & 9 & 15 \\
\hline
\end{tabular}

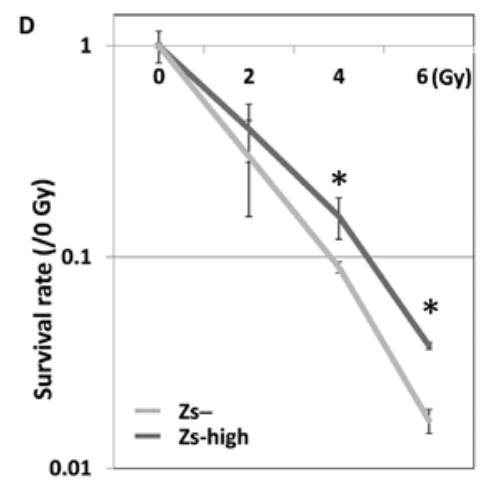

Figure 4. Radiation sensitivities of the fluorescent and non-fluorescent cells. (A and B) Viabilities of (A) TE4 and (B) TE8 cells exposed to radiation at 0 Gy or $8 \mathrm{~Gy}$ after incubation for $120 \mathrm{~h}$. (C and D) Results of clonogenic survival assay in TE8 cells. The number of colonies were counted after incubation for $\sim 2$ weeks (C). Survival rates were analyzed and the results are presented in (C). The error bars represent the standard deviation of the mean for three experiments; asterisks indicate that the mean is significantly different between ZsGreen-high cells and ZsGreen-negative cells (Student's t-test; ${ }^{*} \mathrm{P}<0.05$ ).
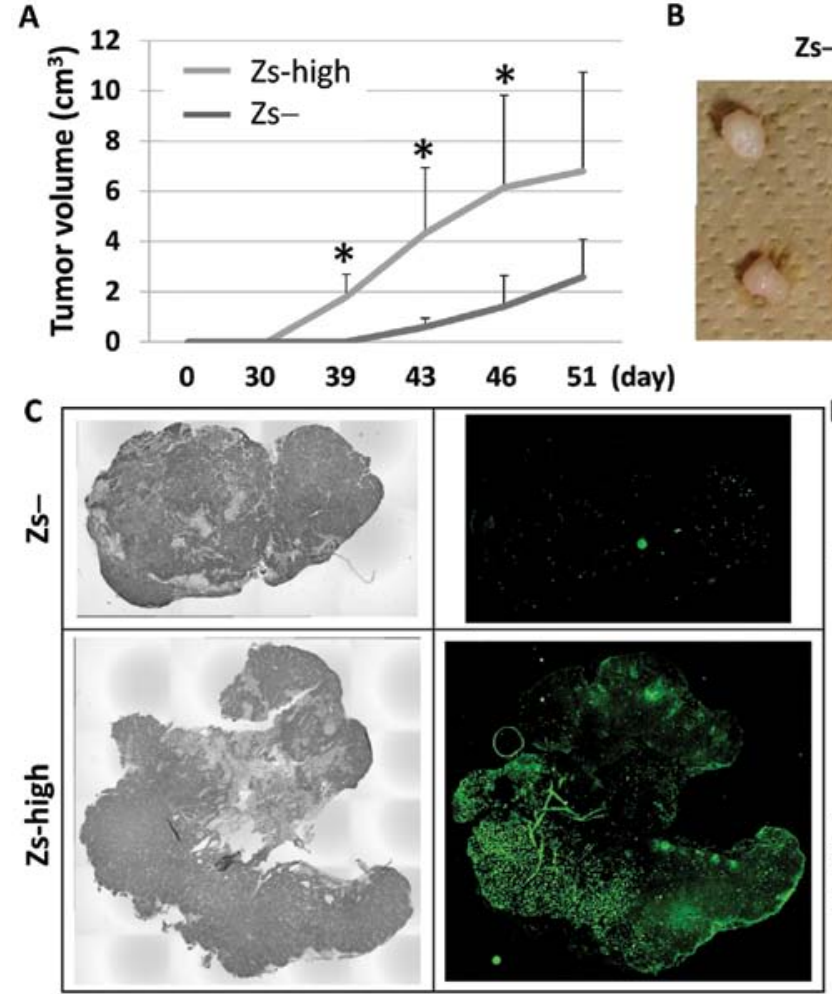

Zs-

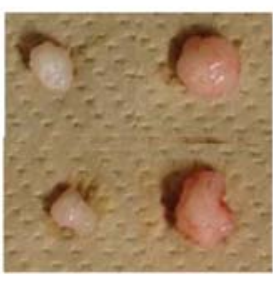

Zs-high
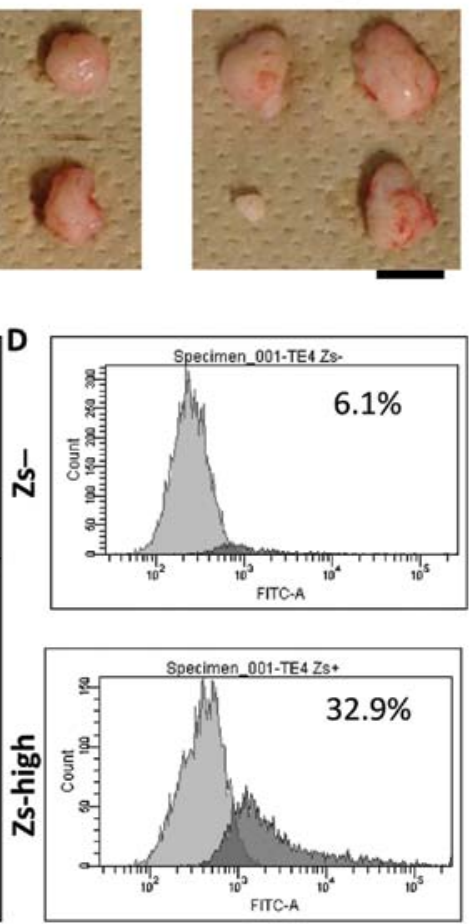

Figure 5. Tumorigenicity study of the fluorescent and non-fluorescent cells in TE4 cells. (A) Tumor volume of xenografts derived from the ZsGreen-high and ZsGreen-negative cells. (B) Formed xenografts derived from the ZsGreen-high and ZsGreen-negative cells in NOD/SCID mice. (C) Xenografts derived from the ZsGreen-high (bottom panel) and ZsGreen-negative cells (upper panel) observed by microscopy. FACS analysis of xenografts derived from the ZsGreenhigh (bottom panel) and ZsGreen-negative cells (upper panel). The error bars represent the standard deviation of the mean of three experiments; the asterisks indicate that the mean is significantly different between ZsGreen-high cells and ZsGreen-negative cells (Student's t-test; "P<0.05). Scale bar, $1 \mathrm{~cm}$.

A higher number of living ZsGreen-high cells than ZsGreennegative cells were observed in TE4 cells (Fig. 4A), but both cell populations were similarly viable in TE8 cells (Fig. 4B).
To further investigate the radiation sensitivities of the ZsGreen-high cells and ZsGreen-negative cells in TE8 cells, clonogenic survival assays were performed. The two popula- 
A

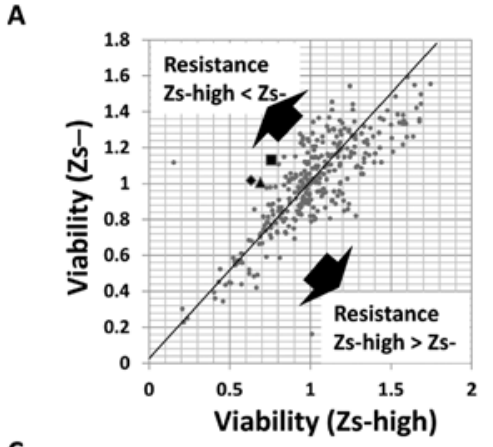

B

\begin{tabular}{|c|c|c|c|}
\hline Viability (Zs-high/Zs-) & \multicolumn{3}{|c|}{ Number } \\
\hline$\sim 0.75$ & 9 & \multirow{2}{*}{22} & \multirow{3}{*}{35} \\
\hline $0.75-0.80$ & 13 & & \\
\hline $0.80-0.85$ & 13 & \multirow{8}{*}{260} & \\
\hline $0.85-0.90$ & 34 & & \multirow{6}{*}{223} \\
\hline $0.90-0.95$ & 38 & & \\
\hline $0.95-1.00$ & 51 & & \\
\hline $1.00-1.05$ & 34 & & \\
\hline $1.05-1.10$ & 45 & & \\
\hline $1.10-1.15$ & 21 & & \\
\hline $1.15-1.20$ & 24 & & \multirow{3}{*}{66} \\
\hline $1.20-1.25$ & 13 & \multirow{2}{*}{42} & \\
\hline $1.25^{\sim}$ & 29 & & \\
\hline
\end{tabular}

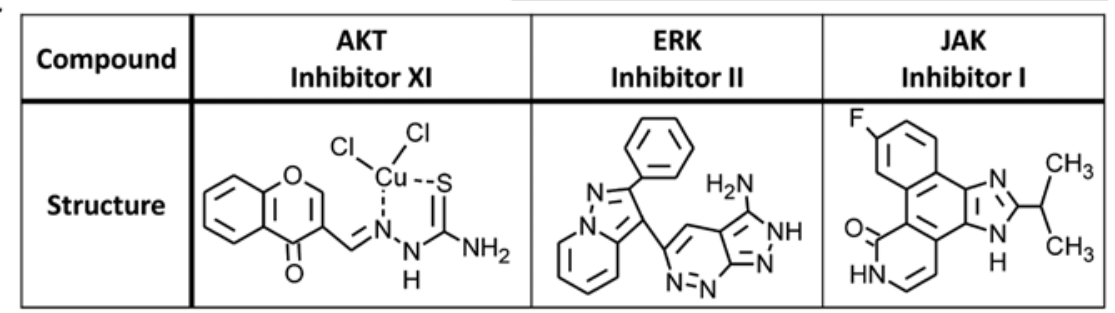

D

TE4

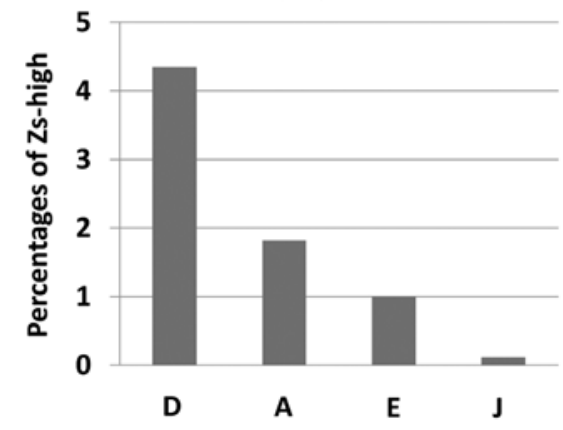

TE8

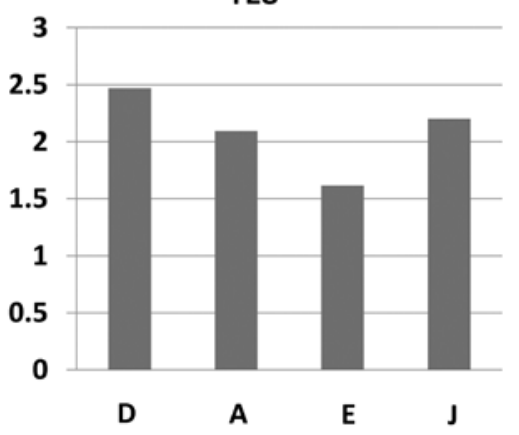

Figure 6. Novel drug discovery for CSC-like cells. (A) Results of drug screening performed with the ZsGreen-high and ZsGreen-negative cells in TE4. (B) Analysis of the results of (A). (C) Three drugs selected in the drug screening. The AKT inhibitor XI is represented as $\mathbf{\Lambda}$, the ERK inhibitor II is represented as $\mathbf{m}$, and the JAK inhibitor I is represented as $\bullet$ in (A). (D) Percentages of Zs-high cells of TE4 and TE8 after treatment with inhibitors. D, DMSO; A, AKT inhibitor XI; E, ERK inhibitor II; J, JAK inhibitor I.

tions isolated in the FACS sorter were seeded in $10-\mathrm{cm}$ dishes and exposed to radiation at $0,2,4$ and 6 Gy. Following incubation for $\sim 2$ weeks, the colonies were counted and the survival rates were analyzed. The number of viable ZsGreen-high cells was more elevated compared with that of the ZsGreen-negative cells in TE8 cells (Fig. 4C and D).

Tumorigenicity study. To investigate the tumorigenicity of the ZsGreen-high and ZsGreen-negative cells, 1x10 ${ }^{3}$ TE4 cells from the two isolated populations were subcutaneously injected into the NOD/SCID mice $(n=4$, each). At day 39, four xenografts derived from the ZsGreen-high cells were detected in the mice, but no xenografts derived from the ZsGreennegative cells were detected. However, four xenografts derived from the ZsGreen-negative cells were detected at day 43 as well (Fig. 5A).

Subsequently, these mice were sacrificed and xenografts were collected (Fig. 5B). Investigation of the sliced sections of xenografts by microscopy and FACS analysis showed that there were several ZsGreen-positive cells in the xenografts derived from the ZsGreen-high cells, but there were few ZsGreen-positive cells in the xenografts derived from the ZsGreen-negative cells (Fig. 5C and D).
The same study should have been performed in TE8, but it was not performed. TE8 was much less tumorigenic than TE4 in NOD/SCID mice. Approximately 500,000 TE8 cells were required to generate xenografts in the mice. In TE8, there were not enough ZsGreen-high cells to provide a sufficient number of fluorescent cells. This was because tumorigenicity studies of these ZsGreen-high and ZsGreen-negative cells in TE8 were not performed.

Drug screening against visualized CSC-like cells. To detect the drugs that can specifically treat the ZsGreen-high cells, drug screening of 324 types of drugs in TE4 cells was performed. After exposure to $10-\mu \mathrm{M}$ concentrations of the drugs for $72 \mathrm{~h}$, the viabilities were analyzed. Consequently, the ZsGreen-high cells were found to exhibit resistance to more drugs compared with that exhibited by the ZsGreen-negative cells (Fig. 6A and B). As a result of the screening, three drugs were selected: AKT inhibitor XI, ERK inhibitor II and JAK inhibitor I (Fig. 6C). To validate the effects of these three drugs, the rate of the ZsGreen-high cells in TE4 and TE8 was analyzed after exposure to each of the drugs with FACS analysis. The drugs were found to kill the ZsGreen-high cells (Fig. 6D). 


\section{Discussion}

The present study revealed that a fluorescent vector consisting of fluorescein ZsGreen fused to cODC could be used to detect CSCs or therapy-resistant cancer cells in esophageal SCC. Transfection into cancer cells with a retroviral vector allowed the populations of ZsGreen-positive and ZsGreennegative cells to be distinguished. The use of FACS sorting made it simple to isolate the populations of ZsGreen-high and ZsGreen-negative cells. Investigation of the characteristics of these two populations indicated that the ZsGreen-high cells were more malignant compared with the ZsGreen-negative cells. The ZsGreen-high cells exhibited higher resistance to CDDP and 5-FU, sphere-forming capacity, and tumorigenicity compared with those exhibited by the ZsGreen-negative cells. In addition, the ZsGreen-high cells survived and proliferated faster (up to $120 \mathrm{~h}$ post-irradiation incubation) compared with the ZsGreen-negative cells in TE4 but not in TE8. The clonogenic survival assay revealed that the ZsGreen-high cells survived longer compared with the ZsGreen-negative cells in TE8. These data suggest that the ZsGreen-high cells are more resistant to radiation compared with the ZsGreen-negative cells.

To detect the drugs targeting the malignant ZsGreen-high cells, drug screening of 324 drugs was performed. AKT inhibitor XI, ERK inhibitor II and JAK inhibitor I were identified as novel drugs targeting the malignant ZsGreen-high cells. AKT is well known as part of the PI3K/AKT pathway, ERK is well known as part of the MEK/ERK pathway and JAK is well known as part of the JAK/STAT pathway. These three pathways are involved in several important phenotypes such as proliferation, differentiation and survival. In the CSCs of several tumor tissues, the PI3K/AKT, MEK/ERK and JAK/STAT pathways are enhanced, and inhibition of the pathways can kill the CSCs in several tumor tissues (14-16). Even in the CSC population of esophageal SCC, the PI3K/AKT pathway was enhanced and inhibition of PI3K or AKT led to reduction in the CSC population (5). These data support the fact that our selected drugs were beneficial in the management of tumor tissues.

In conclusion, a fluorescent vector consisting of fluorescein ZsGreen fused to cODC was used to detect CSCs or therapyresistant cancer cells. In addition, therapy-resistant cells were killed by AKT inhibitor XI, ERK inhibitor II and JAK inhibitor I. These drugs can be used as novel methods in the management of esophageal SCC.

\section{Acknowledgements}

The present study was supported in part by a grant-in-aid for Scientific Research from the Ministry of Education, Culture, Sports, Science and Technology; a grant-in-aid from the Third Comprehensive 10-year Strategy for Cancer Control, Ministry of Health, Labor and Welfare; a grant from the Kobayashi Cancer Research Foundation; a grant from the Princess Takamatsu Cancer Research Fund, Japan; and a grant from the National Institute of Biomedical Innovation. H.I. and M.K. received partial support from Chugai Co., Ltd. and Yakult Honsha Co., Ltd. through institutional endowments.

\section{References}

1. Cheng KK and Day NE: Nutrition and esophageal cancer. Cancer Causes Control 7: 33-40, 1996.

2. Al-Hajj M, Wicha MS, Benito-Hernandez A, et al: Prospective identification of tumorigenic breast cancer cells. Proc Natl Acad Sci USA 100: 3983-3988, 2003.

3. Dembinski JL and Krauss S: Characterization and functional analysis of a slow cycling stem cell-like subpopulation in pancreas adenocarcinoma. Clin Exp Metastasis 26: 611-623, 2009.

4. Tang KH, Dai YD, Tong M, et al: A CD90+ tumor-initiating cell population with an aggressive signature and metastatic capacity in esophageal cancer. Cancer Res 73: 2322-2332, 2013.

5. Li H, Gao Q, Guo L and Lu SH: The PTEN/PI3K/Akt pathway regulates stem-like cells in primary esophageal carcinoma cells. Cancer Biol Ther 11: 950-958, 2011.

6. Zhao JS, Li WJ, Ge D, et al: Tumor initiating cells in esophageal squamous cell carcinomas express high levels of CD44. PLoS One 6: e21419, 2011.

7. Huang SD, Yuan Y, Liu XH, et al: Self-renewal and chemotherapy resistance of p75NTR positive cells in esophageal squamous cell carcinomas. BMC Cancer 9: 9, 2009.

8. Kano Y, Konno M, Ohta K, et al: Jumonji/Arid1b (Jarid1b) protein modulates human esophageal cancer cell growth. Mol Clin Oncol 1: 753-757, 2013.

9. Vlashi E, Kim K, Lagadec C, et al: In vivo imaging, tracking, and targeting of cancer stem cells. J Natl Cancer Inst 101: 350-359, 2009.

10. Pan J, Zhang Q, Wang Y, et al: 26S proteasome activity is downregulated in lung cancer stem-like cells propagated in vitro. PLoS One 5: e13298, 2010.

11. Adikrisna R, Tanaka S, Muramatsu S, et al: Identification of pancreatic cancer stem cells and selective toxicity of chemotherapeutic agents. Gastroenterology 143: 234-245.e7, 2012.

12. Della Donna L, Lagadec C and Pajonk F: Radioresistance of prostate cancer cells with low proteasome activity. Prostate 72: 868-874, 2012.

13. Muramatsu S, Tanaka S, Mogushi K, et al: Visualization of stem cell features in human hepatocellular carcinoma reveals in vivo significance of tumor-host interaction and clinical course. Hepatology 58: 218-228, 2013.

14. Wang YK, Zhu YL, Qiu FM, et al: Activation of Akt and MAPK pathways enhances the tumorigenicity of $\mathrm{CD} 133^{+}$primary colon cancer cells. Carcinogenesis 31: 1376-1380, 2010.

15. Iwanaga R, Wang CA, Micalizzi DS, et al: Expression of Six1 in luminal breast cancers predicts poor prognosis and promotes increases in tumor initiating cells by activation of extracellular signal-regulated kinase and transforming growth factor-beta signaling pathways. Breast Cancer Res 14: R100, 2012.

16. Hernandez-Vargas H, Ouzounova M, Le Calvez-Kelm F, et al: Methylome analysis reveals Jak-STAT pathway deregulation in putative breast cancer stem cells. Epigenetics 6: 428-439, 2011. 